Network structures and temporal stability of self- and informant-rated affective symptoms in Alzheimer's disease

Saari, T. T.

2020-11-01

Saari , T T , Hallikainen , I , Hintsa , T \& Koivisto , A M 2020 , ' Network structures and temporal stability of self- and informant-rated affective symptoms in Alzheimer's disease ' , Journal of Affective Disorders , vol. 276 , pp. 1084-1092 . https://doi.org/10.1016/j.jad.2020.07.100

http://hdl.handle.net/10138/333074

https://doi.org/10.1016/j.jad.2020.07.100

cc_by_nc_nd

acceptedVersion

Downloaded from Helda, University of Helsinki institutional repository.

This is an electronic reprint of the original article.

This reprint may differ from the original in pagination and typographic detail.

Please cite the original version. 


\section{Network structures and temporal stability of self- and informant-rated affective}

\section{symptoms in Alzheimer's disease}

Saari $\mathrm{TT}^{12^{*}}$, Hallikainen $\mathrm{I}^{1}$, Hintsa $\mathrm{T}^{2}$, Koivisto $\mathrm{AM}^{1345}$

${ }^{1}$ Department of Neurology, University of Eastern Finland, Kuopio, Finland

${ }^{2}$ School of Educational Sciences and Psychology, University of Eastern Finland, Joensuu,

Finland

${ }^{3}$ Department of Neurology, Kuopio, Kuopio University Hospital, Finland

${ }^{4}$ Department of Neurosciences, University of Helsinki, Helsinki, Finland

${ }^{5}$ Department of Internal Medicine and Rehabilitation, Helsinki University Hospital, Helsinki,

Finland

This manuscript has been published in final, edited form at Journal of Affective Disorders. Please cite as:

Saari, T. T., Hallikainen, I., Hintsa, T., \& Koivisto, A. M. (2020). Network structures and temporal stability of self- and informant-rated affective symptoms in Alzheimer's disease. Journal of Affective Disorders, 276, 1084-1092. https://doi.org/10.1016/j.jad.2020.07.100

Background: Affective symptoms in Alzheimer's disease (AD) can be rated with both informant- and self-ratings. Information from these two modalities may not converge. We estimated network structures of affective symptoms in AD with both rating modalities and assessed the longitudinal stability of the networks.

\footnotetext{
Corresponding Author:

Toni Saari

Yliopistonranta 1B,

FIN-70210 Kuopio, Finland

Phone: +358 503259130

toni.saari@uef.fi
} 
Methods: Network analyses combining self-rated and informant-rated affective symptoms were conducted in 3198 individuals with $A D$ at two time points (mean follow-up 387 days), drawn from the NACC database. Self-rated symptoms were assessed by Geriatric Depression Scale, and informant-rated symptoms included depression, apathy and anxiety questions from Neuropsychiatric Inventory Questionnaire.

Results: Informant-rated symptoms were mainly connected to symptoms expressing lack of positive affect, but not to the more central symptoms of self-rated worthlessness and helplessness. Networks did not differ in structure $(p=0.71)$, or connectivity $(p=0.92)$ between visits. Symptoms formed four clinically meaningful clusters of depressive symptoms and decline, lack of positive affect, informant-rated apathy and anxiety and informant-rated depression.

Limitations: The symptom dynamics in our study could have been present before AD diagnosis. The lack of positive affect cluster may represent a methodological artefact rather than a theoretically meaningful subgroup. Requiring follow-up lead to a selection of patients with less cognitive decline.

Conclusions: Informant rating may only capture the more visible affective symptoms, such as not being in good spirits, instead of more central and severe symptoms, such as hopelessness and worthlessness. Future research should continue to be mindful of differences between self- and informant-rated symptoms even in earlier stages of AD.

Keywords: Network analysis, Alzheimer's disease, depression, apathy, anxiety, neuropsychiatric symptoms Running head: Affective symptom networks in AD 
Published as a preprint: https://psyarxiv.com/7jwsk 


\section{Introduction}

Affective symptoms like apathy, depression, and anxiety are some of the most common psychological disturbances in Alzheimer's disease (AD; Zhao et al., 2016). While these symptoms may not be severe enough to warrant a formal diagnosis, they often co-occur (Cummings et al., 1994; Levy et al., 1998; Teri et al., 1999), persist or resurface later (Olin et al., 2002; Vik-Mo et al., 2018) and associate with worse quality of life (Hongisto et al., 2018) as well as performance of activities of daily living (Palmer et al., 2011). Affective symptoms are likely multifactorial (Lanctôt et al., 2017; Marin, 1991), for example, it has been postulated that depressive symptoms could reflect the individual's reaction to declining cognition (Fitz \& Teri, 1994; Weintraub, Xie, Karlawish, \& Siderowf, 2007), whereas apathy has been associated with neurobiological changes, such as disturbances in frontal circuitry (Rosenberg et al., 2015).

Both self-report and informant-report are used when assessing neuropsychiatric symptoms. The measures are often implicitly considered to reflect the same underlying construct, although the information derived from them may diverge (Georgi, Vlckova, Lukavsky, Kopecek, \& Bares, 2018; Olin et al., 2002; Teri \& Wagner, 1992), at least partially owing to anosognosia (Robert et al., 2018) and caregiver characteristics (de Vugt et al., 2004). Additionally, measures thought to reflect one construct, i.e. depression, may also include items relevant for another construct, i.e. apathy (Levy et al., 1998).

In addition to utilizing different sources of information, majority of the research on affective symptoms in $A D$ is conducted using total scores of self- or informant-rated measures, masking the contribution of individual symptoms, such as feeling worthless, to the psychopathological picture of AD (e.g. Olin et al., 2002). Interpretation of summary scores is 
challenging, as they may combine symptoms of varying etiologies and clinical importance. Therefore, it is valuable to examine the relationships between the individual symptoms themselves, instead of assuming them to be caused by any single construct, such as depression. It is also important to track individual symptoms to determine whether they predict developing, more widespread psychopathology (Robert et al., 2018).

Relationships between individual symptoms can be investigated using network analysis (Borsboom \& Cramer, 2013), a method that has been widely adopted in psychopathology research covering psychiatric disorders (longitudinally, e.g. van Borkulo et al., 2015; von Stockert, Fried, Armour, \& Pietrzak, 2018). Recently, network structures of depressive symptoms have been analyzed in a general geriatric setting (van Wanrooij et al., 2019) as well.

In this study, we use network analysis to investigate how both self- and informant-rated affective symptoms relate to one another in Alzheimer's disease in a longitudinal research design. By combining both sources of information and symptom-level data, we can examine the relative importance of individual affective symptoms in $A D$ and estimate the extent to which these two information sources converge on a symptom level. Stability of symptom networks over two visits is also studied.

\section{Methods}

\subsection{Participants and measures}

Data for this study were obtained from the University of Washington's National Alzheimer's Coordinating Center (NACC) that aims to facilitate research in the field by sharing data. The NACC's Uniform Data Set (UDS), consisting of individuals with normal cognition, mild cognitive impairment or dementia stage neurodegenerative disorder (Weintraub et al., 
2018) was used in this study. Participants were recruited to Alzheimer's Disease Centers (ADCs), and underwent standardized comprehensive cognitive, behavioral and functional evaluation. Informed consent was acquired in written form from study patients and informants.

In this study, we used data from 39 ADC's, and the UDS visits were conducted between September 2005 and February 2019. From the UDS, we first selected individuals with a diagnosis of $A D$. The diagnosis of $A D$ was made in accordance with the National Institute of Neurological and Communicative Diseases and Stroke/Alzheimer's Disease and Related Disorders Association criteria for probable Alzheimer's disease (McKhann et al., 1984) criteria until a revision of the UDS protocol in 2015 (Morris et al., 2006). After the 2015 revision, the National Institute on Aging-Alzheimer's Association criteria for AD were used (McKhann et al., 2011; Besser et al., 2018).

In addition to a diagnosis of $A D$, age $\geq 65$ years, and complete data on self- and informantratings of depression, anxiety and apathy at two visits were required. Of the initial sample of 7581 individuals with $A D$ and self- and informant-rating data and at least 65 years of age, 3679 participants had follow-up data for the same measures and were further explored. State-of-the-art outlier removal method of median absolute deviation (MAD; Leys et al., 2013) was used to exclude participants clearly outside the approximately annual follow-up, leaving a final sample of 3198 individuals with AD. As a robustness check, all analyses included in this study have also been performed in the sample without outlier removal, and included in Supplementary Materials.

\subsubsection{Self-rated affective symptoms}

For self-reported affective symptoms, we used Geriatric Depression Scale (GDS; Yesavage et al., 1982; Yesavage \& Sheikh, 1986), one of the most common depression screening instruments for aged populations. We further divided GDS to an apathy subscale, GDS-3A 
(Adams et al., 2004; van Wanrooij et al., 2019), consisting of "dropped activities", "feeling full of energy" (reverse-scored) and "prefer to stay at home", and a depression subscale, GDS-12D, consisting of the remaining 12 items.

\subsubsection{Informant-rated affective symptoms}

For informant-rated affective symptoms, we used depression, apathy and anxiety questions of the Neuropsychiatric Inventory Questionnaire (NPI-Q, Kaufer et al., 2000). NPI-Q is an abbreviated version of the widely used Neuropsychiatric Inventory (Cummings et al., 1994), tapping into various psychopathological disturbances typically observed in neurodegenerative disorders. We included apathy and anxiety questions, as GDS has items related to these constructs. Additionally, NPI apathy and anxiety have loaded on the same factor as depression, although not consistently (Canevelli et al., 2013). Apathy should be conceptually distinct from depression (Levy et al., 1998), however, the previously identified GDS-3A suggests there are apathy items in the GDS (Adams et al., 2004; Kim et al., 2013; van Wanrooij et al., 2019). Anxiety is a common comorbidity with depression in AD (Teri et al., 1999), and factor analyses of GDS have demonstrated the presence of anxiety items (Adams et al., 2004), such as being afraid something bad might happen.

\subsubsection{Cognitive and functional measures}

Participants underwent standardized neuropsychological assessment designed to assess all major cognitive domains, although the specific tests in the protocol were updated during the study period (Morris et al., 2006; Weintraub et al., 2018). To characterize the study sample, we present data on only measures of global cognition. Global cognition was assessed using either Mini-Mental State Examination (MMSE; Folstein et al., 1975), a brief instrument for global cognitive screening, or Montreal Cognitive Assessment (MoCA; 
Nasreddine et al., 2005), a similar instrument that has been favored over MMSE in recent years. Whether MoCA or MMSE was used depended on the form version used at the study visit; those with a more recent visit have undergone MoCA assessment. For more details regarding the revision of the neuropsychological assessment used in the UDS, see Weintraub et al. (2018). Disease severity was assessed with the $\mathrm{CDR}^{\circledR}$ Dementia Staging Instrument (CDR; Hughes et al., 1982), in which a score of 0.5 corresponds to mild cognitive impairment or very mild AD, 1 to mild, 2 to moderate and 3 to severe dementia. In our study, the CDR scores reflected AD severity.

\subsection{Statistical analysis}

All statistical analyses were conducted in R version 3.5.3 (R Core Team, 2019). Code for the analyses is included in the Supplementary Materials and at osf.io/njvsa/.

\subsubsection{Differences related to follow-up}

Differences between individuals who had only baseline data $(n=3902)$ versus individuals who had also follow-up data, and differences between baseline versus follow-up data were analysed using t-tests and chi-square tests. As comparisons of large groups are prone to find statistically significant differences between many given variables (Meehl, 1990; Orben \& Przybylski, 2019), Cohen's d was used as an effect size estimate of these differences.

\subsubsection{Network estimation}

Network analysis is used to reveal variable interactions within phenomena of interest, such as in psychiatric disorders (Borsboom \& Cramer, 2013). Networks can serve as an alternative to factor analytic models (Bringmann \& Eronen, 2018; Fried, 2015), which may carry with them problematic causal assumptions (Borsboom et al., 2003), i.e. latent entity 
"depression" causing "worthlessness". In networks, symptom relationships are visualized as partial correlations, indicating the number and strength of unique associations (later, edges) any individual symptom, (later, node) has with other nodes. This framework allows for a thorough investigation of how symptoms interact, in line with clinical understanding of symptoms, and suggesting which symptoms may be the most crucial in psychiatric disturbances (Fried \& Nesse, 2015).

Networks of 15 GDS symptoms and 3 NPI-Q symptoms were estimated using Ising models, a novel method for network analysis of binary data (van Borkulo et al., 2015). Briefly, the method uses logistic regressions of each variable regressed on all others, and Least Absolute Shrinkage and Selection Operator (LASSO; Tibshirani, 1996) to construct a sparse model, where small edges are set to zero. Ising models were constructed using R package IsingFit (van Borkulo, 2016), and visualized using qgraph (Epskamp et al., 2012). For a comprehensive introduction to Ising networks and their use in psychopathology research, see van Borkulo et al. (2015).

\subsubsection{Network inference}

We used $m g m$ package (Haslbeck \& Waldorp, 2016) to estimate how well other nodes in the network could predict the presence of a node. For binary data, the predictability estimate is normalized correct classification (nCC), indicated in our study by the blue circle around each node (Haslbeck \& Waldorp, 2018). Nodes high in nCC are highly predictable based on the presence of the neighboring nodes, whereas low nCC indicates relative independence. In some instances, the nCC estimate may be negative due to over-fitting (Haslbeck \& Waldorp, 2018). For graphical arguments the nCC of these near-zero nodes were set to exactly zero (no blue circle around the node). 
Strength estimates of individual nodes were also analysed (Epskamp et al., 2018). Strength is a node centrality estimate, depicting how many and/or strong connections each individual node has. Instead of drawing inferences on the visual inspection of network structures themselves, centrality indices serve to quantify the relative importance of individual nodes. We used z-scores instead of raw scores in our study to facilitate comparison of strength estimates at two time points.

\subsubsection{Network robustness}

Bootstrapping methods were applied to edge weights and strength to estimate their robustness, using bootnet (Epskamp et al., 2018).

\subsubsection{Network comparison}

Invariance of the two network structures, global strengths and individual edges can be analysed using NetworkComparisonTest package (NCT; van Borkulo, 2016). However, if network structures do not differ significantly, examining individual edge differences may inflate the risk of type I error and should be avoided (Borkulo et al., 2017). Thus, only network structure and global strength invariances were analysed.

\subsubsection{Community detection}

Finally, we examined community structures of the networks at two time points. A community is a group of nodes that have dense connections inside the group, but sparse connections to other groups (Newman \& Girvan, 2004). We used two community detection methods from igraph (Csardi \& Nepusz, 2006), first of which is based on walktrap algorithm (Pons \& Latapy, 2005), an agglomerative approach that detects the central nodes of a community with ease, but may be ambiguous in peripheral node detection (Newman \& 
Girvan, 2004). The second method is based on spinglass algorithm (Reichardt \& Bornholdt, 2006) derived from principles of statistical mechanics. As spinglass produces slightly different results every time, 100 iterations were ran and a solution equivalent with the median number of communities in these iterations is presented. Results of walktrap community detection are presented in this paper, and spinglass results are located in the Supplementary Materials.

3. Results

\subsection{Sample characteristics}

Clinical and demographic data of participants is presented in Table 1. Of 3679 individuals, 481 were excluded due to them having a shorter than 259 or longer than 518 days of followup, as defined by MAD, leaving us the analytic sample of 3198 individuals. Participants with follow-up data were younger, more educated, had higher MMSE, MoCA, CDR and CDR-SOB scores and lower GDS scores than participants who had only baseline data (all $p^{\prime} s<.05$ ). However, the differences ranged from very small to medium in effect size (Cohen's d $0.04-$ 0.37), highest for MMSE and lowest for age. 
Table 1. Characteristics of the study sample.

Baseline Follow-up

Mean/\% SD Mean/\% SD

Demographics

Age, years

$77.5 \quad 6.7$

Gender, female

52.8

Ethnicity, Caucasian ${ }^{a}$

83.8

Education, years ${ }^{b}$

14.4

3.7

Clinical characteristics

Cognitive Testing: MMSE $(0-30)^{c}$

$\begin{array}{llll}21.8 & 4.4 & 20.0 & 5.3\end{array}$

Cognitive Testing: MoCA $(0-30)^{c}$

$\begin{array}{llll}15.8 & 5.2 & 13.8 & 6.1\end{array}$

GDS Total Score (0-15)

$\begin{array}{llll}2.3 & 2.4 & 2.1 & 2.4\end{array}$

CDR Sum of Boxes (0-18)

$\begin{array}{llll}5.3 & 2.8 & 6.8 & 3.4\end{array}$

CDR Global Score (0-3)

$\begin{array}{llll}0.9 & 0.5 & 1.2 & 0.6\end{array}$

Proportion positive for symptom

NPI-Q Depression/Dysphoria

$\begin{array}{llll}0.37 & 0.48 & 0.34 & 0.47\end{array}$

NPI-Q Anxiety/Nervousness

$\begin{array}{llll}0.35 & 0.48 & 0.36 & 0.48\end{array}$

NPI-Q Apathy/Indifference

$\begin{array}{llll}0.39 & 0.49 & 0.42 & 0.49\end{array}$

GDS Satisfied with Life*

$\begin{array}{llll}0.11 & 0.31 & 0.10 & 0.30\end{array}$

GDS Dropped Activities and Interests

$\begin{array}{llll}0.29 & 0.45 & 0.25 & 0.43\end{array}$

GDS Feel That Life is Empty

$\begin{array}{llll}0.09 & 0.29 & 0.10 & 0.30\end{array}$

GDS Often Get Bored

$\begin{array}{llll}0.17 & 0.37 & 0.17 & 0.37\end{array}$ 


\begin{tabular}{|c|c|c|c|}
\hline GDS In Good Spirits Most of the Time* & 0.06 & 0.24 & 0.05 \\
\hline GDS Afraid Something Bad is Going to Happen & 0.09 & 0.29 & 0.07 \\
\hline GDS Feel Happy Most of the Time* & 0.09 & 0.28 & 0.08 \\
\hline GDS Often Feel Helpless & 0.14 & 0.34 & 0.13 \\
\hline GDS Prefer to Stay at Home & 0.26 & 0.44 & 0.24 \\
\hline GDS More Memory Problems than Most & 0.47 & 0.50 & 0.42 \\
\hline GDS Wonderful to be Alive* & 0.04 & 0.20 & 0.04 \\
\hline GDS Feel Pretty Worthless & 0.10 & 0.30 & 0.10 \\
\hline GDS Feel Full of Energy* & 0.27 & 0.45 & 0.25 \\
\hline GDS Feel Situation is Hopeless & 0.07 & 0.25 & 0.07 \\
\hline GDS Think Most People Are Better Off & 0.08 & 0.28 & 0.08 \\
\hline
\end{tabular}

Note. * Items have been reverse scored, $\mathrm{a}=$ missing data from 6 inviduals, $\mathrm{b}=$ missing data from 11 individuals. C, Participants were administered either MMSE or MoCA: MMSE for 2913 and 2844, MoCA for 237 and 300, cognitive data were missing from 48 and 51 participants, for baseline and follow-up, respectively. Ranges for clinical measures are included in parentheses. GDS = Geriatric Depression Scale, $\mathrm{CDR}=$ Clinical Dementia Rating, $\mathrm{MMSE}=$ Mini-Mental State Examination, $\mathrm{MoCA}=$ Montreal Cognitive Assessment, NPI-Q = Neuropsychiatric Inventory - Questionnaire

The mean time between visits was $386.74(\mathrm{SD}=48.1$ ) days. At follow-up, participants performed worse in cognitive testing on MMSE $(t(2834)=-28.63, p<.001, d=0.39)$ and $\operatorname{MoCA}(t(231)=8.55, p<.001, d=0.35)$, had higher CDR global score $(t(3179)=-38.01, p<$ $.001, d=0.43)$ and CDR-SOB $(t(3179)=-38.01, p<.001, d=0.48)$, but scored slightly lower 
on GDS $(t(3179)=5.01, p<.001, d=0.08)$ compared to baseline. At both time points, GDS item "More Memory Problems Than Most" was the most highly endorsed, whereas the item "Wonderful to Be Alive" (reverse-scored) was the least endorsed. Informant-rated affective symptoms, measured with NPI-Q, were for the most part more frequently observed than participant-rated symptoms.

\subsection{Symptom networks}

Figure 1 shows the networks at both time points. Relatively strong connections were observed between node 10 "In Good Spirits Most of the Time" and node 12 "Feel Happy Most of the Time", as well as node 8 "Feel that Life is Empty" and node 9 "Often Get Bored". Normalized correct classification, indicating how well a node is predicted by neighboring nodes, was highest for node 12 "Feel Happy Most of the Time" (0.2 and 0.21), but was relatively low averaged over all nodes $(M=0.10, S D=0.06$ and $M=0.11, S D=0.06)$ at baseline and follow-up, respectively. For both networks, node 11 "Afraid Something Bad is Going to Happen" was not predicted by other nodes in the network, and node 18 "Think Most People are Better Off" was not meaningfully predicted by other nodes. Notable crossmodal connections were observed between informant-rated depression and (lack of) selfrated happy mood or being in good spirits.

Figure 2 indicates how many and/or strong connections each node had with other nodes. Highly similar strength estimates were observed at baseline and at follow-up, although satisfaction with life seemed to be more connected at follow-up. NPI-Q item for depressive symptoms was widely connected, however NPI-Q apathy item did not have strong connections with GDS-3A apathy questions despite conceptual resemblance. Additionally, NPI-Q anxiety item was not connected outside the NPI-Q triad. Self-reported memory 
problem was also a relatively isolated node, as was staying at home and being afraid that something bad might happen. Feeling helpless or worthless, or not feeling happy, however, seemed to be more central symptoms.

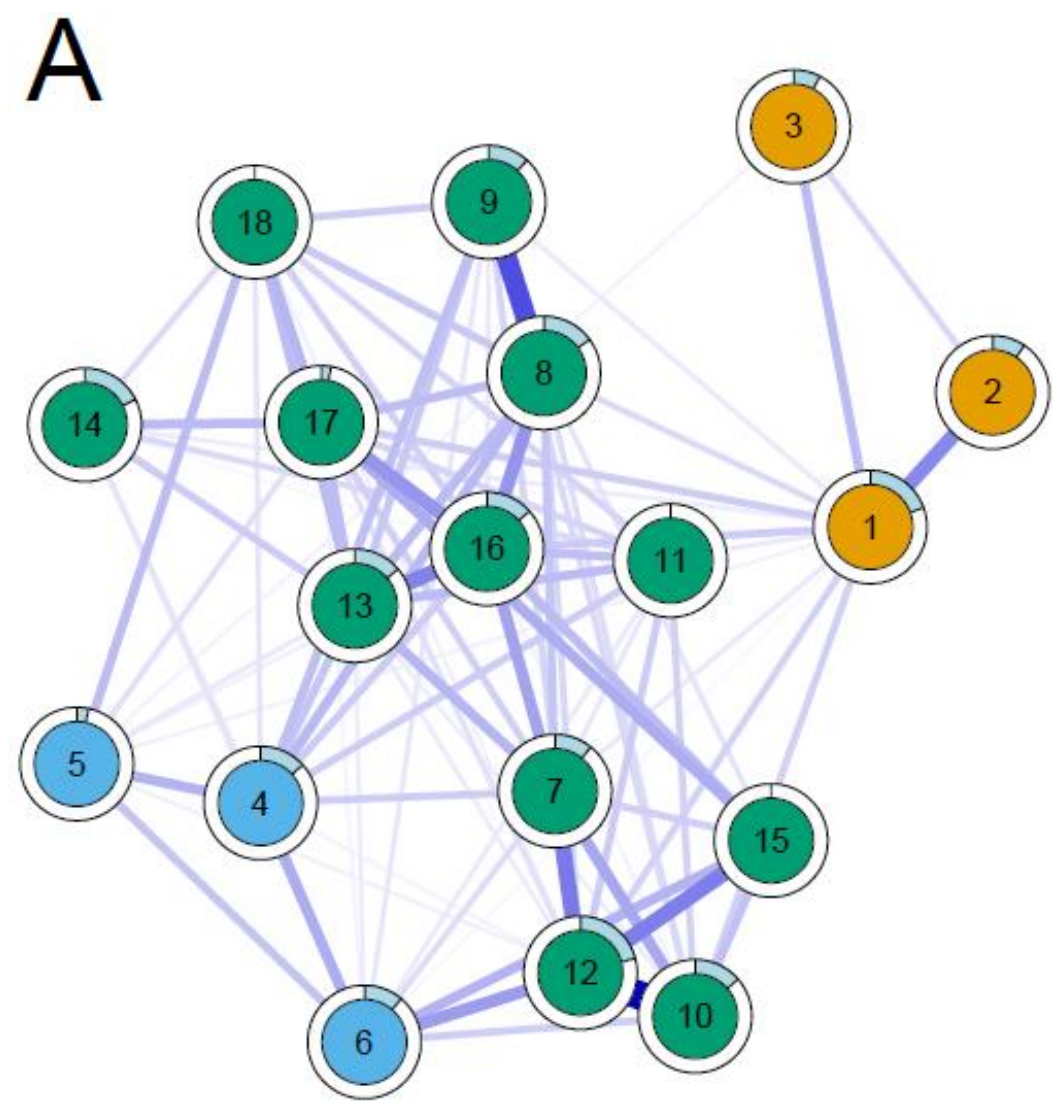




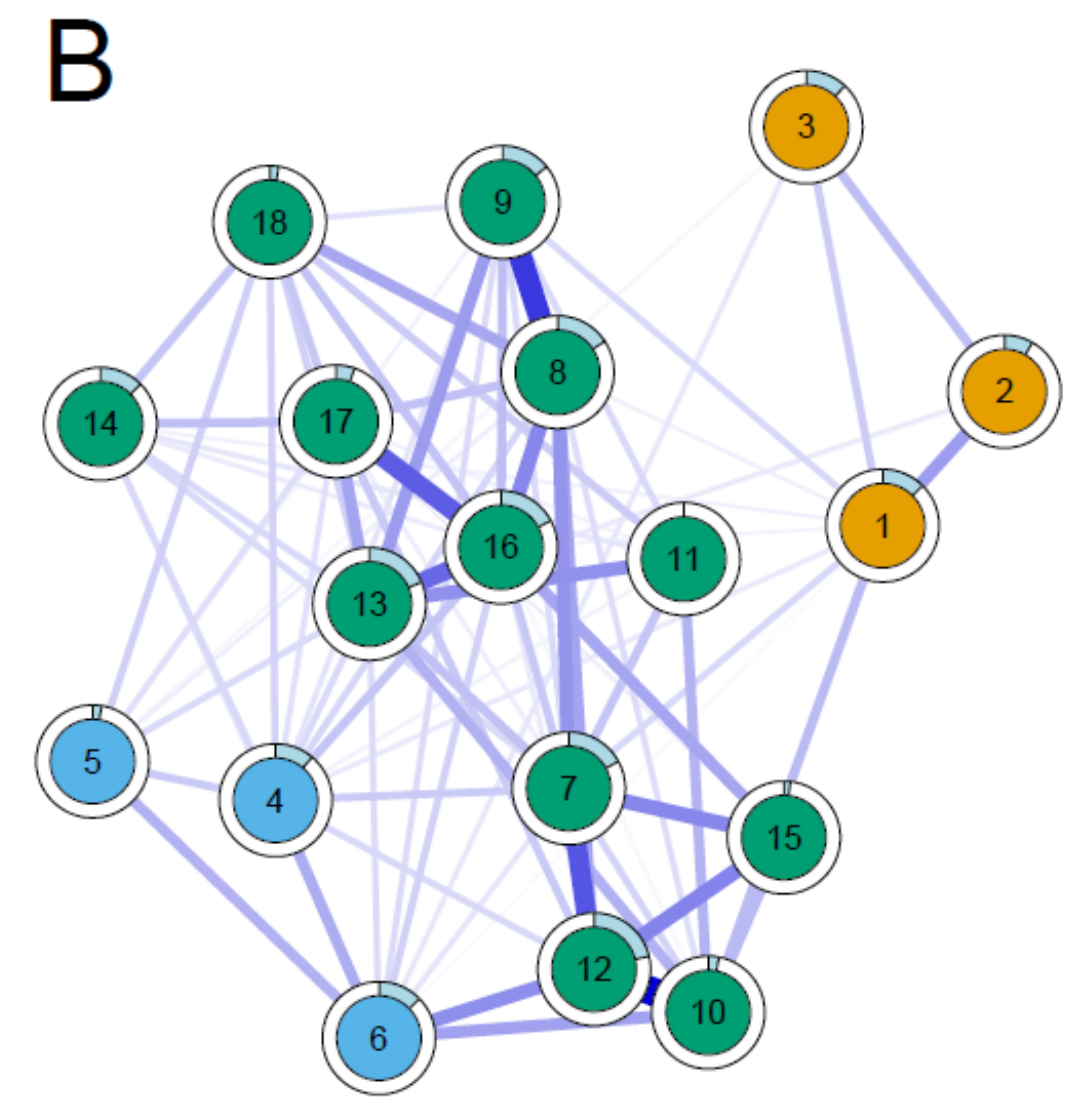

NPI-Q

- 1: Sad or Depressed

- 2: Nervous

- 3: Less Interested GDS-3A

- 4: Dropped Activities

- 5: Stay at Home

- 6: Full of Energy >< GDS-12D

- 7: Satisfied with Life ><

- 8: Life is Empty

- 9: Bored

- 10: Good Spirits ><

- 11: Afraid

- 12: Happy ><

- 13: Helpless

- 14: Memory Problems

- 15: Wonderful to be Alive ><

- 16: Worthless

- 17: Hopeless

- 18: Most People Better Off

Figure 1. Network structures at baseline (A) and at follow-up (B). Orange nodes represent NPI-Q symptoms, as reported by an informant. Green nodes represent the twelve GDS items thought to assess depressive symptoms, and blue nodes correspond to the three GDS items related to apathy. Edges, or blue lines between the nodes, denote unique connections when conditioning for all other nodes in the network (van Borkulo et al., 2015), where thicker edges denote stronger connections. Blue circles around the nodes depict the degree of normalized correct classification, which is an index of predictability for binary data above what is trivially predicted by the relative probability of given condition (symptom present or not) irrespective of other nodes (Haslbeck \& Waldorp, 2018). Layout of the network is averaged over the two visits, and reverse-scored items are indicated by $><$ in the legend. 


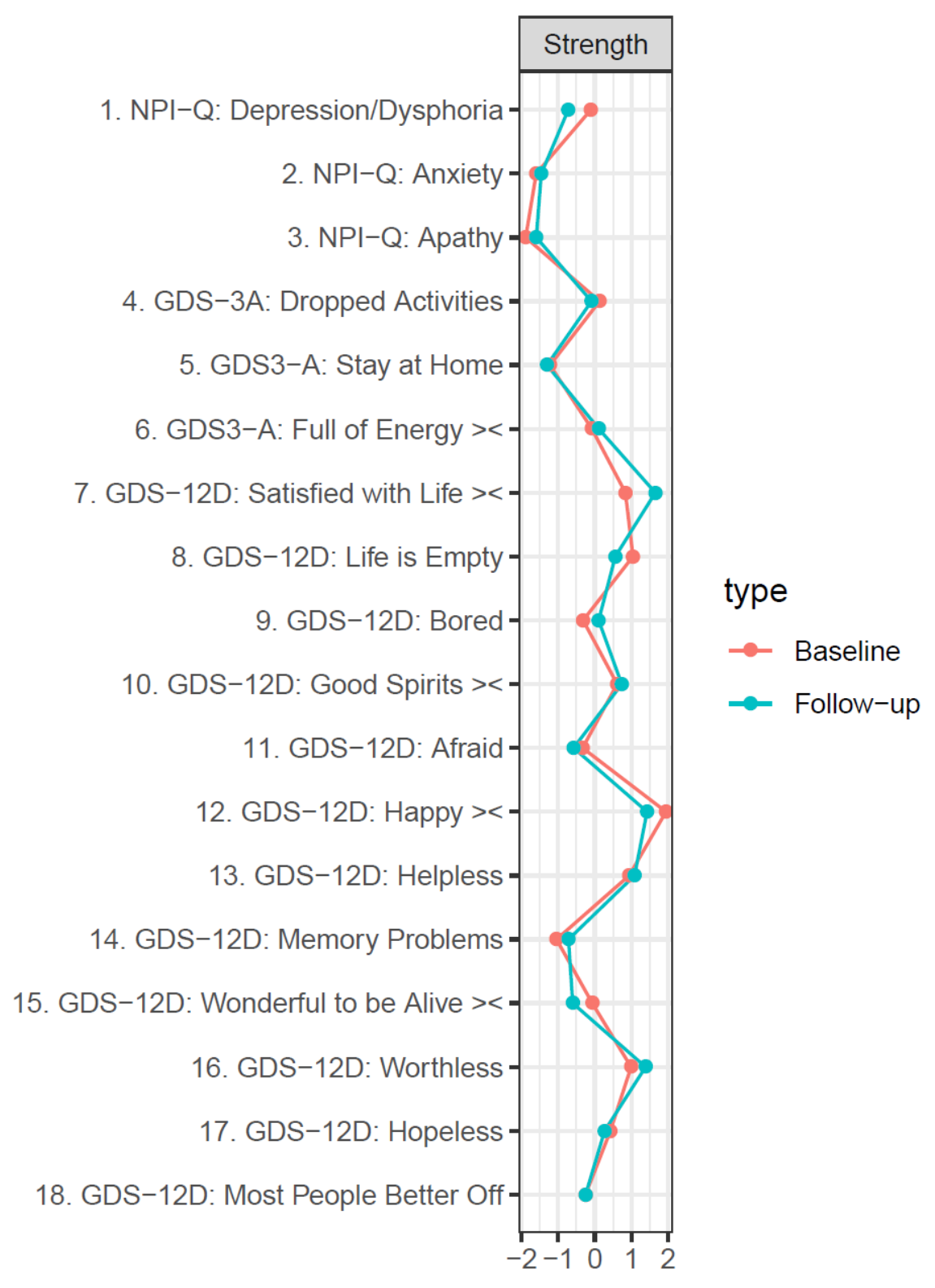

Figure 2. Standardized strength estimates of the nodes at baseline and at follow-up.

Strength is a centrality measure for networks, which indicates the direct connectedness of a node (Epskamp et al., 2018). Strength values are the summary of edge weights connecting to a node, and these values were standardized for comparison in the figure.

Bootstrapping methods were used to analyse whether networks, edge weights and strength estimates are robust and inferences justified (Supplementary Materials, Figures S1-S8). Of 
note, correlation stability of the strength coefficient in Figure 2 were .67 and .75 at baseline and follow-up, respectively, indicating that the strength estimates are reliable (over the recommended cut-off of .5, Epskamp et al., 2018).

The networks were compared statistically to see if they differed between baseline and follow-up. No difference was found in network structure $(0.71, p=0.59)$, nor in global strength $(0.92, p=0.63)$.

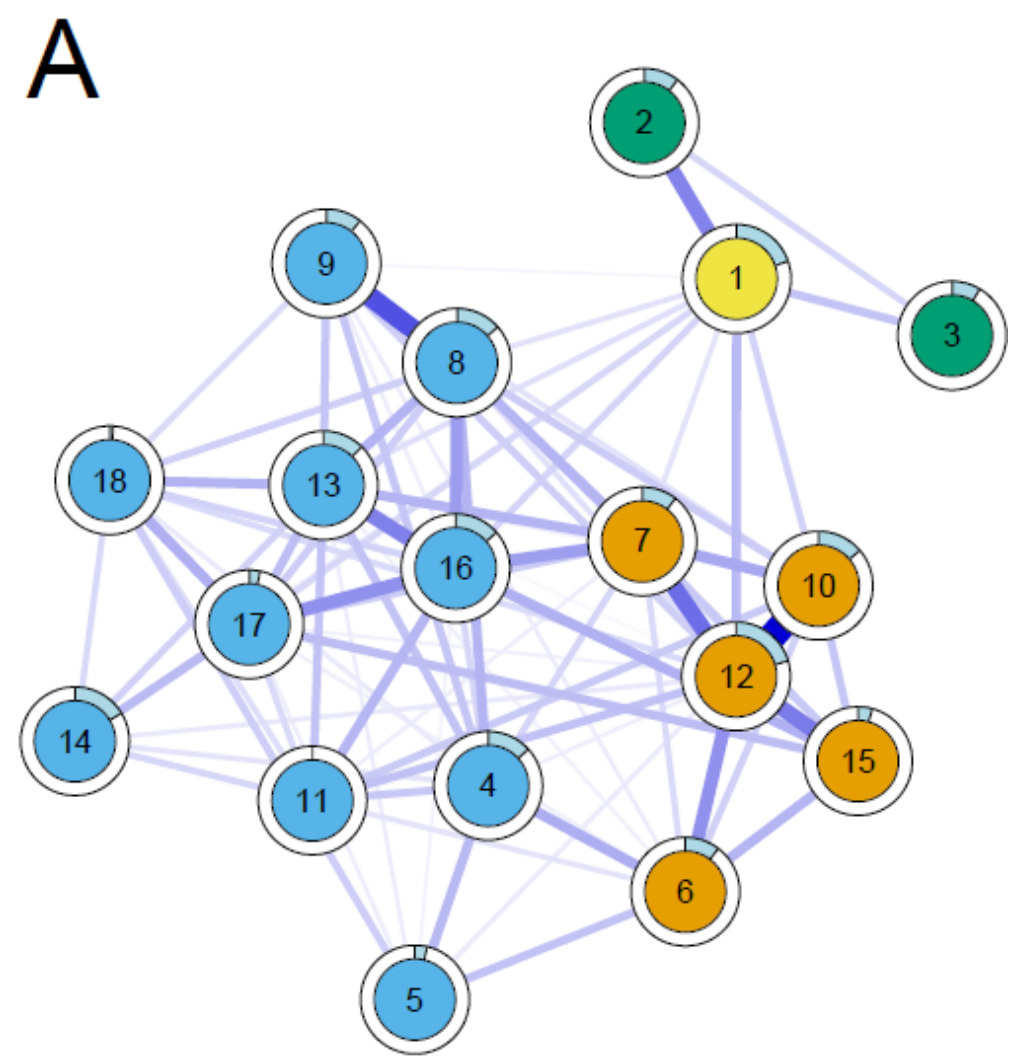




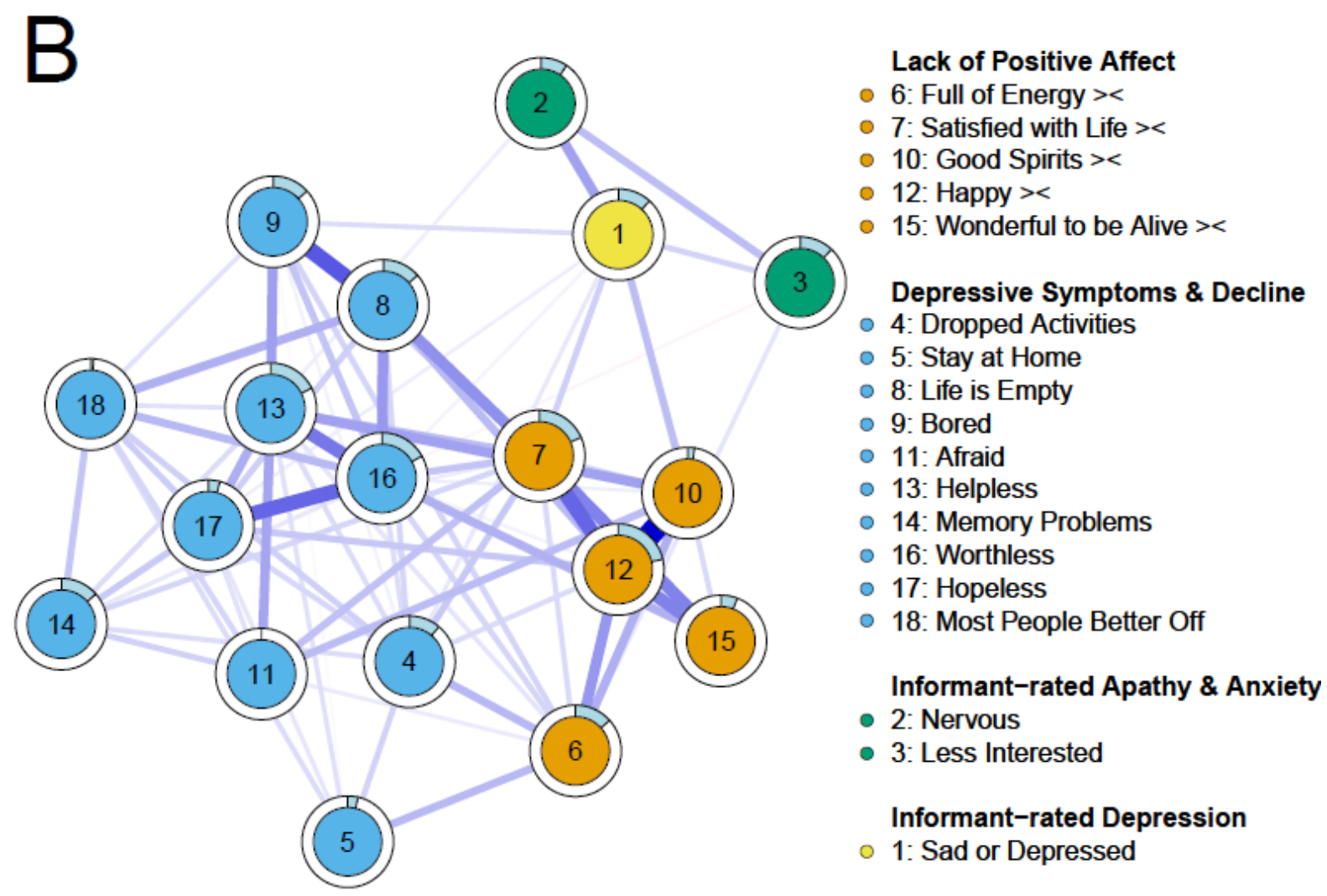

Figure 3. Community detection labelled networks using walktrap algorithm at baseline (A)

and follow-up (B), where colors represent membership of communities. Note the discrepancies between a priori divisions in Figure 1 and this figure. Orange nodes represent the five reverse-scored items, interpreted as lack of positive affect. Blue nodes include depressive symptoms and symptoms that relate to decreasing capabilities, green nodes depict informant-rated apathy and anxiety, and the yellow node represents the one-node community of informant-rated depression.

Figure 3 demonstrates the results of community detection via walktrap algorithm. Notably, the community structures were identical at both time points (but not for spinglass method, see Figure S9, where a five-community structure was found for follow-up). Four communities were established, where the largest community represents depressive symptoms in combination with symptoms related to decreasing capabilities, a two-node 
community includes informant-rated apathy and anxiety, and a five-node community denoting lack of positive affect. Finally, the last community is represented by just the informant-rated depression, bridging self-rated symptoms and other informant-rated symptoms.

None of the communities included a solution where informant-rated depressive symptoms would be included with self-rated depressive symptoms. Only spinglass results at follow-up supported the GDS-3A category.

All analyses were also performed without MAD outlier removal for time between visits, and highly similar results were found in terms of network structure, comparison between baseline and follow-up and centrality of individual nodes (Supplementary Materials). Community detection solutions were identical in the two samples.

\section{Discussion}

The aims of the study were to investigate network structures of affective symptoms in $A D$ rated by the participant and an informant, and to examine whether the network structures were longitudinally stable. We found affective symptom networks to be stable across two visits in a large sample of elderly individuals with $A D$. Four communities of lack of positive affect, depressive symptoms and declining capabilities, informant-rated rated apathy and anxiety, and informant-rated depression were found. The most central symptoms in the first two large communities were not feeling happy for lack of positive affect, and feelings of worthlessness and helplessness for the depressive community. The informant-rated depression question had many, but relatively weak, connections to self-rated depression symptoms, perhaps capturing only the most visible, not necessarily the most central symptoms. Informant-rated symptoms of anxiety and apathy, also content represented in 
the GDS questions, were mostly associated with informant-rated depression but not selfrated symptoms of similar content. Communities of self-rated symptoms differed slightly according to community detection method used, reflecting possible difficulties in assigning a symptom to just one community.

Stability of networks across the follow-up is in line with a recent study demonstrating that majority of the variance over time in GDS could be accounted for by a stable trait in elderly individuals (Gana et al., 2017). Network analytic research is just taking its first steps in neurodegenerative disease context, however the networks discovered in our study are markedly similar to those found recently in a sample of aged persons, largely without a diagnosis of dementia (van Wanrooij et al., 2019). Furthermore, a longitudinal study indicated that GDS-15 depressive symptoms, apart from memory problems, are similarly reported in individuals who reach CDR .5 and in those who remain at CDR 0 (Masters et al., 2015). Thus, there seems to be tentative evidence for symptom-level overlap in affective symptom dynamics in aged persons, with or without Alzheimer's disease.

Particularly, the similar connections between symptoms with a 'desperate quality' (Adams et al., 2004), such as helplessness, hopelessness and worthlessness, were found to be at the core of the symptom networks. We extended findings of van Wanrooij et al. by showing that informant-rated depressive symptoms are rather weakly connected to severe symptoms, such as hopelessness, which is a diagnosis-independent risk factor for suicide (Beck et al., 1990; Fried \& Nesse, 2015). In line with van Wanrooij et al. (2019), we found anxiety (worry something bad might happen) and memory impairment to be weakly connected to other symptoms. Additionally, we found similar ambivalence regarding whether lack of energy was an indicator of absence of 'positive mood' (Kim et al., 2013) or apathy. 
Furthermore, we found mixed evidence to support the GDS-3A apathy subscale, as both community detection methods would identify dropping of activities and preference to stay at home as part of broader depressive community, with the exception of spinglass method producing the apathetic symptom triad only at follow-up. This need not be controversial, as it may be reasonable to assume that one node can belong to multiple communities (Reichardt \& Bornholdt, 2006): for example, having dropped activities may be related to several plausible causal chains, whether connected to depressive or apathetic symptoms (Marin, 1991). Hypothetically, we can formulate at least the following: dropping activities due to loss of interest, dropping activities due to burden of several depressive symptoms, or dropping activities due to cognitive impairment and being distressed by this loss. Based on symptom-level analysis, it is evident that the border between apathetic versus depressive disorders is fuzzy and the symptoms are perhaps more meaningfully modelled by networks, where sharp categories are not expected (Borsboom \& Cramer, 2013; Fried, 2015; van Wanrooij et al., 2019).

The findings of this study may have clinical relevance. For example, it is acknowledged that informant reports may be biased in terms of severity, but our findings suggest that, owing to their nature, informant questionnaires could also be biased towards visible affective symptoms. Symptoms such as hopelessness or worthlessness could be crucial to understanding the patient's experience, yet these symptoms may be underappreciated in informant questionnaires (Mograbi \& Morris, 2014). Notably, these are the same symptoms that are considered useful in differentiating apathy from depression (Tagariello et al., 2009). In light of our data, research on affective symptoms in AD seems complex. Our findings highlight the limitations in using summary scores of GDS-15, GDS-12D or GDS-3A to denote 
a symptom or a syndrome a priori (Marin, 1991; Fried, 2015; Fried \& Nesse, 2015).

Furthermore, it appears that informant-rated depressive symptoms only tap into some of the self-reported symptoms. Discrepancies between self- and informant ratings are likely influenced by several factors, such as differences in brevity and content of self and informant questions, anosognosia (Robert et al., 2018), biases in perceiving oneself versus others (Pronin, 2008; Allik et al., 2010), caregiver characteristics (de Vugt et al., 2004; Pfeifer et al., 2013) and variable representations of 'normal' age-related changes in mood (Georgi et al., 2018).

\subsection{Limitations}

Our study had some limitations. First, it is possible that the individuals enrolled in this study were already exhibiting similar patterns of affective symptoms before AD diagnosis, explaining similarities between networks found in this study and that found previously in predominantly cognitively healthy elderly (van Wanrooij et al., 2019). This issue could be further explored by constructing similar networks longitudinally in at-risk or $\mathrm{MCl}$ samples, who were later diagnosed with AD. However, as network analytic literature is still sparse in $A D$ research, we considered characterization and temporal analysis of symptom dynamics to be a relevant opening for further studies. Second, a meta-analysis of the factor structure of GDS suggested that the language-invariant co-occurrence of the five reverse-scored items related to positive mood may simply reflect a methodological artefact, rather than a theoretically substantive clustering (Kim et al., 2013). However, network analysis allows us to demonstrate the connections inside and outside this five-node community, not just that they cluster together. 
Third, our decision to remove individuals with very long or very short follow-up periods reduced the sample size. However, we consider this justifiable, as our analyses now represent more clearly an annual follow-up, and thus may generalize better to clinical contexts as well. It is also important to keep in mind that the network analytic methods here are considered to reveal the true network structure in sample sizes this large even with outlier removal (van Borkulo et al., 2015). Indeed, supplementary analyses revealed that our results are not dependent on outlier removal. Finally, the dropout rate was substantial and individuals who continued in the study were younger, more educated, cognitively and functionally less impaired and had lower total GDS-15 scores. These features, while not unusual in longitudinal studies of individuals with $A D$, may limit the external validity of our results.

\subsection{Strengths}

To our knowledge, this is the first, large-scale network analysis of affective symptoms in $A D$ using data from two rating modalities. Our results complement factor analytic literature by showing that investigating detailed symptom relationships is a valid approach to model psychopathology in at least the early stages of AD. We were also able to demonstrate temporal stability of these symptom networks in a clinically relevant follow-up interval of one year. Furthermore, we demonstrated how informant-rated depressive symptoms align variably with self-rated symptoms, deepening the understanding of discrepancies between rating modalities. Finally, robustness analyses strengthened the validity of our models.

\subsection{Conclusions}

Networks of affective symptoms in individuals with AD were highly stable across a year of follow-up. Feelings of worthlessness and helplessness were central symptoms at both time 
points, but they were relatively independent of informant-rated depressive symptoms. Informant-rated depressive symptoms were mostly connected to symptoms conveying lack of positive affect. No connections were found between informant-rated apathy and selfrated apathetic symptoms. Future research should continue to be mindful of differences between self- and informant-rated symptoms even in earlier stages of $A D$, and further utilize symptom-level data to increase precision in diagnostics and clinical intervention.

\section{Acknowledgements}

The NACC database is funded by NIA/NIH Grant U01 AG016976. NACC data are contributed by the NIA-funded ADCs: P30 AG019610 (PI Eric Reiman, MD), P30 AG013846 (PI Neil Kowall, MD), P50 AG008702 (PI Scott Small, MD), P50 AG025688 (PI Allan Levey, MD, PhD), P50 AG047266 (PI Todd Golde, MD, PhD), P30 AG010133 (PI Andrew Saykin, PsyD), P50 AG005146 (PI Marilyn Albert, PhD), P50 AG005134 (PI Bradley Hyman, MD, PhD), P50 AG016574 (PI Ronald Petersen, MD, PhD), P50 AG005138 (PI Mary Sano, PhD), P30 AG008051 (PI Thomas Wisniewski, MD), P30 AG013854 (PI Robert Vassar, PhD), P30 AG008017 (PI Jeffrey Kaye, MD), P30 AG010161 (PI David Bennett, MD), P50 AG047366 (PI Victor Henderson, MD, MS), P30 AG010129 (PI Charles DeCarli, MD), P50 AG016573 (PI Frank LaFerla, PhD), P50 AG005131 (PI James Brewer, MD, PhD), P50 AG023501 (PI Bruce Miller, MD), P30 AG035982 (PI Russell Swerdlow, MD), P30 AG028383 (PI Linda Van Eldik, PhD), P30 AG053760 (PI Henry Paulson, MD, PhD), P30 AG010124 (PI John Trojanowski, MD, PhD), P50 AG005133 (PI Oscar Lopez, MD), P50 AG005142 (PI Helena Chui, MD), P30 AG012300 (PI Roger Rosenberg, MD), P30 AG049638 (PI Suzanne Craft, PhD), P50 AG005136 (PI Thomas Grabowski, MD), P50 AG033514 (PI Sanjay Asthana, MD, FRCP), P50 AG005681 (PI John Morris, MD), P50 AG047270 (PI Stephen Strittmatter, MD, PhD). 
We wish to thank UEF Neurology and Brain Research Unit for providing facilities to carry out this research.

Funding

TS was supported by grants from the Finnish Brain Foundation and the Finnish Cultural Foundation. 


\section{References}

Adams, K. B., Matto, H. C., \& Sanders, S. (2004). Confirmatory Factor Analysis of the Geriatric Depression Scale. The Gerontologist, 44(6), 818-826.

https://doi.org/10.1093/geront/44.6.818

Allik, J., Realo, A., Mõttus, R., Borkenau, P., Kuppens, P., \& Hřebíčková, M. (2010). How people see others is different from how people see themselves: A replicable pattern across cultures. Journal of Personality and Social Psychology, 99(5), 870-882. https://doi.org/10.1037/a0020963

Beck, A. T., Brown, G., Berchick, R. J., Stewart, B. L., \& Steer, R. A. (1990). Relationship Between Hopelessness and Ultimate Suicide: A Replication with Psychiatric Outpatients. The American Journal of Psychiatry, 147(2), 190-195.

Besser, L., Kukull, W., Knopman, D. S., Chui, H., Galasko, D., Weintraub, S., Jicha, G., Carlsson, C., Burns, J., Quinn, J., Sweet, R. A., Rascovsky, K., Teylan, M., Beekly, D., Thomas, G., Bollenbeck, M., Monsell, S., Mock, C., Zhou, X. H., ... Morris, J. C. (2018). Version 3 of the National Alzheimer's Coordinating Center's Uniform Data Set. Alzheimer Disease \& Associated Disorders, 1-8. https://doi.org/10.1097/WAD.0000000000000279

Borsboom, D., \& Cramer, A. O. J. (2013). Network Analysis: An Integrative Approach to the Structure of Psychopathology. Annual Review of Clinical Psychology, 9(1), 91-121. https://doi.org/10.1146/annurev-clinpsy-050212-185608

Borsboom, D., Mellenbergh, G. J., \& van Heerden, J. (2003). The theoretical status of latent variables. Psychological Review, 110(2), 203-219. https://doi.org/10.1037/0033-295X.110.2.203

Bringmann, L. F., \& Eronen, M. I. (2018). Don't blame the model: Reconsidering the network approach to psychopathology. Psychological Review, 125(4), 606-615. https://doi.org/10.1037/rev0000108

Canevelli, M., Adali, N., Voisin, T., Soto, M. E., Bruno, G., Cesari, M., \& Vellas, B. (2013). Behavioral and psychological subsyndromes in Alzheimer's disease using the Neuropsychiatric 
Inventory: Behavioral subsyndromes in Alzheimer's disease. International Journal of Geriatric Psychiatry, 28(8), 795-803. https://doi.org/10.1002/gps.3904

Csardi, G., \& Nepusz, T. (2006). The igraph software package for complex network research. InterJournal, Complex Systems, 1-9.

Cummings, J. L., Mega, M., Gray, K., Rosenberg-Thompson, S., Carusi, D. A., \& Gornbein, J. (1994). The Neuropsychiatric Inventory: Comprehensive assessment of psychopathology in dementia. Neurology, 44(12), 2308-2308. https://doi.org/10.1212/WNL.44.12.2308

de Vugt, M. E., Stevens, F., Aalten, P., Lousberg, R., Jaspers, N., Winkens, I., Jolles, J., \& Verhey, F. R. J. (2004). Do caregiver management strategies influence patient behaviour in dementia? International Journal of Geriatric Psychiatry, 19(1), 85-92. https://doi.org/10.1002/gps.1044 Epskamp, S., Borsboom, D., \& Fried, E. I. (2018). Estimating psychological networks and their accuracy: A tutorial paper. Behavior Research Methods, 50(1), 195-212. https://doi.org/10.3758/s13428-017-0862-1

Epskamp, S., Cramer, A. O. J., Waldorp, L. J., Schmittmann, V. D., \& Borsboom, D. (2012). qgraph: Network Visualizations of Relationships in Psychometric Data. Journal of Statistical Software, 48(4). https://doi.org/10.18637/jss.v048.i04

Fitz, A. G., \& Teri, L. (1994). Depression, Cognition, and Functional Ability in Patients with Alzheimer's Disease. Journal of the American Geriatrics Society, 42(2), 186-191. https://doi.org/10.1111/j.1532-5415.1994.tb04950.x

Folstein, M. F., Folstein, S. E., \& McHugh, P. R. (1975). “Mini-mental state”. A practical method for grading the cognitive state of patients for the clinician. Journal of Psychiatric Research, 12(3), 189-198. https://doi.org/10.1016/0022-3956(75)90026-6

Fried, E. I. (2015). Problematic assumptions have slowed down depression research: Why symptoms, not syndromes are the way forward. Frontiers in Psychology, 6. https://doi.org/10.3389/fpsyg.2015.00309 
Fried, E. I., \& Nesse, R. M. (2015). Depression sum-scores don't add up: Why analyzing specific depression symptoms is essential. BMC Medicine, 13(1). https://doi.org/10.1186/s12916015-0325-4

Gana, K., Bailly, N., Broc, G., Cazauvieilh, C., \& Boudouda, N. E. (2017). The Geriatric Depression Scale: Does it measure depressive mood, depressive affect, or both? International Journal of Geriatric Psychiatry, 32(10), 1150-1157. https://doi.org/10.1002/gps.4582

Georgi, H. S., Vlckova, K. H., Lukavsky, J., Kopecek, M., \& Bares, M. (2018). Beck Depression Inventory-II: Self-report or interview-based administrations show different results in older persons. International Psychogeriatrics, 1-8. https://doi.org/10.1017/S1041610218001187

Haslbeck, J.M.B., Waldorp, L.J. (2016). mgm: Structure Estimation for Time-Varying Mixed Gr aphical Models in high-dimensional Data arXiv preprint:1510.06871v2 URL http://arxiv.org/a bs/1510.06871v2.

Haslbeck, J. M. B., \& Waldorp, L. J. (2018). How well do network models predict observations? On the importance of predictability in network models. Behavior Research Methods, 50(2), 853861. https://doi.org/10.3758/s13428-017-0910-x

Hongisto, K., Hallikainen, I., Selander, T., Törmälehto, S., Väätäinen, S., Martikainen, J., Välimäki, T., Hartikainen, S., Suhonen, J., \& Koivisto, A. M. (2018). Quality of Life in relation to neuropsychiatric symptoms in Alzheimer's disease: 5-year prospective ALSOVA cohort study: QoL in relation to NPS in Alzheimer's disease. International Journal of Geriatric Psychiatry, 33(1), 47-57. https://doi.org/10.1002/gps.4666

Hughes, C. P., Berg, L., Danziger, W. L., Coben, L. A., \& Martin, R. L. (1982). A new clinical scale for the staging of dementia. The British Journal of Psychiatry, 140(6), 566-572. https://doi.org/10.1192/bjp.140.6.566

Kaufer, D. I., Cummings, J. L., Ketchel, P., Smith, V., MacMillan, A., Shelley, T., Lopez, O. L., \& DeKosky, S. T. (2000). Validation of the NPI-Q, a Brief Clinical Form of the Neuropsychiatric 
Inventory. The Journal of Neuropsychiatry and Clinical Neurosciences, 12(2), 233-239. https://doi.org/10.1176/jnp.12.2.233

Kim, G., DeCoster, J., Huang, C.-H., \& Bryant, A. N. (2013). A meta-analysis of the factor structure of the Geriatric Depression Scale (GDS): The effects of language. International Psychogeriatrics, 25(1), 71-81. https://doi.org/10.1017/S1041610212001421

Lanctôt, K. L., Amatniek, J., Ancoli-Israel, S., Arnold, S. E., Ballard, C., Cohen-Mansfield, J., Ismail, Z., Lyketsos, C., Miller, D. S., Musiek, E., Osorio, R. S., Rosenberg, P. B., Satlin, A., Steffens, D., Tariot, P., Bain, L. J., Carrillo, M. C., Hendrix, J. A., Jurgens, H., \& Boot, B. (2017). Neuropsychiatric signs and symptoms of Alzheimer's disease: New treatment paradigms. Alzheimer's \& Dementia: Translational Research \& Clinical Interventions, 3(3), 440-449. https://doi.org/10.1016/j.trci.2017.07.001

Levy, M. L., Cummings, J. L., Fairbanks, L. A., Masterman, D., Miller, B. L., Craig, A. H., Paulsen, J. S., \& Litvan, I. (1998). Apathy Is Not Depression. The Journal of Neuropsychiatry and Clinical Neurosciences, 10(3), 314-319. https://doi.org/10.1176/jnp.10.3.314

Leys, C., Ley, C., Klein, O., Bernard, P., \& Licata, L. (2013). Detecting outliers: Do not use standard deviation around the mean, use absolute deviation around the median. Journal of Experimental Social Psychology, 49(4), 764-766. https://doi.org/10.1016/j.jesp.2013.03.013

Marin, R. S. (1991). Apathy: A Neuropsychiatric Syndrome. The Journal of Neuropsychiatry and Clinical Neurosciences, 3, 243-254.

Masters, M. C., Morris, J. C., \& Roe, C. M. (2015). “Noncognitive” symptoms of early Alzheimer disease: A longitudinal analysis. Neurology, 84(6), 617-622. https://doi.org/10.1212/WNL.0000000000001238

McKhann, G., Drachman, D., Folstein, M., Katzman, R., Price, D., \& Stadlan, E. M. (1984). Clinical diagnosis of Alzheimer's disease: Report of the NINCDS-ADRDA Work Group* under the auspices of Department of Health and Human Services Task Force on Alzheimer's Disease. Neurology, 34(7), 939-939. https://doi.org/10.1212/WNL.34.7.939 
McKhann, G. M., Knopman, D. S., Chertkow, H., Hyman, B. T., Jack, C. R., Kawas, C. H., Klunk, W. E., Koroshetz, W. J., Manly, J. J., Mayeux, R., Mohs, R. C., Morris, J. C., Rossor, M. N., Scheltens, P., Carrillo, M. C., Thies, B., Weintraub, S., \& Phelps, C. H. (2011). The diagnosis of dementia due to Alzheimer's disease: Recommendations from the National Institute on AgingAlzheimer's Association workgroups on diagnostic guidelines for Alzheimer's disease. Alzheimer's \& Dementia, 7(3), 263-269. https://doi.org/10.1016/j.jalz.2011.03.005 Meehl, P. E. (1990). Why Summaries of Research on Psychological Theories are Often Uninterpretable. Psychological Reports, 66(1), 195-244. https://doi.org/10.2466/pr0.1990.66.1.195

Mograbi, D. C., \& Morris, R. G. (2014). On the Relation among Mood, Apathy, and Anosognosia in Alzheimer's Disease. Journal of the International Neuropsychological Society, 20(1), 2-7. https://doi.org/10.1017/S1355617713001276

Morris, J. C., Weintraub, S., Chui, H. C., Cummings, J., DeCarli, C., Ferris, S., Foster, N. L., Galasko, D., Graff-Radford, N., Peskind, E. R., Beekly, D., Ramos, E. M., \& Kukull, W. A. (2006). The Uniform Data Set (UDS): Clinical and Cognitive Variables and Descriptive Data From Alzheimer Disease Centers: Alzheimer Disease \& Associated Disorders, 20(4), 210-216. https://doi.org/10.1097/01.wad.0000213865.09806.92

Nasreddine, Z. S., Phillips, N. A., Bédirian, V., Charbonneau, S., Whitehead, V., Collin, I., Cummings, J. L., \& Chertkow, H. (2005). The Montreal Cognitive Assessment, MoCA: A Brief Screening Tool For Mild Cognitive Impairment. Journal of the American Geriatrics Society, 53(4), 695-699. https://doi.org/10.1111/j.1532-5415.2005.53221.x

Newman, M. E. J., \& Girvan, M. (2004). Finding and evaluating community structure in networks. Physical Review E, 69(2), 026113. https://doi.org/10.1103/PhysRevE.69.026113

Olin, J. T., Katz, I. R., Meyers, B. S., Schneider, L. S., \& Lebowitz, B. D. (2002). Provisional Diagnostic Criteria for Depression of Alzheimer Disease: Rationale and Background. The American 
Journal of Geriatric Psychiatry, 10(2), 129-141. https://doi.org/10.1097/00019442-

200203000-00004

Orben, A., \& Przybylski, A. K. (2019). The association between adolescent well-being and digital technology use. Nature Human Behaviour, 3(2), 173-182. https://doi.org/10.1038/s41562018-0506-1

Palmer, K., Lupo, F., Perri, R., Salamone, G., Fadda, L., Caltagirone, C., Musicco, M., \& Cravello, L. (2011). Predicting Disease Progression in Alzheimer's Disease: The Role of Neuropsychiatric Syndromes on Functional and Cognitive Decline. Journal of Alzheimer's Disease, 24(1), 3545. https://doi.org/10.3233/JAD-2010-101836

Pfeifer, L., Drobetz, R., Fankhauser, S., Mortby, M. E., Maercker, A., \& Forstmeier, S. (2013). Caregiver rating bias in mild cognitive impairment and mild Alzheimer's disease: Impact of caregiver burden and depression on dyadic rating discrepancy across domains. International Psychogeriatrics, 25(8), 1345-1355. https://doi.org/10.1017/S1041610213000562

Pons, P., \& Latapy, M. (2005). Computing communities in large networks using random walks (long version). ArXiv:Physics/0512106. http://arxiv.org/abs/physics/0512106

Pronin, E. (2008). How We See Ourselves and How We See Others. Science, 320(5880), 1177-1180. https://doi.org/10.1126/science.1154199

R Core Team. (2019). R: A Language and Environment for Statistical Computing (3.5.3) [Computer software]. R Foundation for Statistical Computing. https://www.R-project.org/

Reichardt, J., \& Bornholdt, S. (2006). Statistical mechanics of community detection. Physical Review E, 74(1), 016110. https://doi.org/10.1103/PhysRevE.74.016110

Robert, P., Lanctôt, K. L., Agüera-Ortiz, L., Aalten, P., Bremond, F., Defrancesco, M., Hanon, C., David, R., Dubois, B., Dujardin, K., Husain, M., König, A., Levy, R., Mantua, V., Meulien, D., Miller, D., Moebius, H. J., Rasmussen, J., Robert, G., ... Manera, V. (2018). Is it time to revise the diagnostic criteria for apathy in brain disorders? The 2018 international consensus group. European Psychiatry, 54, 71-76. https://doi.org/10.1016/j.eurpsy.2018.07.008 
Rosenberg, P. B., Nowrangi, M. A., \& Lyketsos, C. G. (2015). Neuropsychiatric symptoms in Alzheimer's disease: What might be associated brain circuits? Molecular Aspects of Medicine, 43-44, 25-37. https://doi.org/10.1016/j.mam.2015.05.005

Tagariello, P., Girardi, P., \& Amore, M. (2009). Depression and apathy in dementia: Same syndrome or different constructs? A critical review. Archives of Gerontology and Geriatrics, 49(2), 246249. https://doi.org/10.1016/j.archger.2008.09.002

Teri, L., Ferretti, L. E., Gibbons, L. E., Logsdon, R. G., McCurry, S. M., Kukull, W. A., McCormick, W. C., Bowen, J. D., \& Larson, E. B. (1999). Anxiety in Alzheimer's Disease: Prevalence and Comorbidity. The Journals of Gerontology Series A: Biological Sciences and Medical Sciences, 54(7), M348-M352. https://doi.org/10.1093/gerona/54.7.M348

Teri, L., \& Wagner, A. (1992). Alzheimer's disease and depression. Journal of Consulting and Clinical Psychology, 60(3), 379-391. https://doi.org/10.1037/0022-006X.60.3.379

Tibshirani, R. (1996). Regression Shrinkage and Selection via the Lasso. Journal of the Royal Statistical Society. Series B (Methodological), 58(1), 267-288.

van Borkulo, C., Boschloo, L., Borsboom, D., Penninx, B. W. J. H., Waldorp, L. J., \& Schoevers, R. A. (2015). Association of Symptom Network Structure With the Course of Depression. JAMA Psychiatry, 72(12), 1219. https://doi.org/10.1001/jamapsychiatry.2015.2079

van Borkulo, C., Epskamp, S., with contributions from Robitzsch, A. (2016). IsingFit: Fitting Isi ng Models Using the ELasso Method. R package version 0.3.1. https://CRAN.R-project.org/p ackage=IsingFit

van Borkulo C.D., with contributions from Epskamp, S. and Millner, A. (2016). NetworkComp arisonTest: Statistical Comparison of Two Networks Based on Three Invariance Measures. R package version 2.0.1. https://CRAN.R-project.org/package =NetworkComparisonTest 
van Borkulo, C., Boschloo, L., Kossakowski, J. J., Tio, P., Schoevers, R., Borsboom, D., \& Waldorp, L. (2017). Comparing network structures on three aspects: A permutation test. https://doi.org/10.13140/rg.2.2.29455.38569

van Borkulo, C. D., Borsboom, D., Epskamp, S., Blanken, T. F., Boschloo, L., Schoevers, R. A., \& Waldorp, L. J. (2015). A new method for constructing networks from binary data. Scientific Reports, 4(1), 5918. https://doi.org/10.1038/srep05918

van Wanrooij, L. L., Borsboom, D., Moll van Charante, E. P., Richard, E., \& van Gool, W. A. (2019). A network approach on the relation between apathy and depression symptoms with dementia and functional disability. International Psychogeriatrics, 1-9. https://doi.org/10.1017/S1041610218002387

Vik-Mo, A. O., Giil, L. M., Ballard, C., \& Aarsland, D. (2018). Course of neuropsychiatric symptoms in dementia: 5-year longitudinal study. International Journal of Geriatric Psychiatry, 33(10), 1361-1369. https://doi.org/10.1002/gps.4933

von Stockert, S. H. H., Fried, E. I., Armour, C., \& Pietrzak, R. H. (2018). Evaluating the stability of DSM5 PTSD symptom network structure in a national sample of U.S. military veterans. Journal of Affective Disorders, 229, 63-68. https://doi.org/10.1016/j.jad.2017.12.043

Weintraub, D., Xie, S., Karlawish, J., \& Siderowf, A. (2007). Differences in depression symptoms in patients with Alzheimer's and Parkinson's diseases: Evidence from the 15-item Geriatric Depression Scale (GDS-15). International Journal of Geriatric Psychiatry, 22(10), 1025-1030. https://doi.org/10.1002/gps.1785

Weintraub, S., Besser, L., Dodge, H. H., Teylan, M., Ferris, S., Goldstein, F. C., Giordani, B., Kramer, J., Loewenstein, D., Marson, D., Mungas, D., Salmon, D., Welsh-Bohmer, K., Zhou, X.-H., Shirk, S. D., Atri, A., Kukull, W. A., Phelps, C., \& Morris, J. C. (2018). Version 3 of the Alzheimer Disease Centers' Neuropsychological Test Battery in the Uniform Data Set (UDS): Alzheimer Disease \& Associated Disorders, 32(1), 10-17.

https://doi.org/10.1097/WAD.0000000000000223 
Yesavage, J. A., Brink, T. L., Rose, T. L., Lum, O., Huang, V., Adey, M., \& Leirer, V. O. (1982).

Development and validation of a geriatric depression screening scale: A preliminary report. Journal of Psychiatric Research, 17(1), 37-49. https://doi.org/10.1016/0022-3956(82)900334

Yesavage, J. A., \& Sheikh, J. I. (1986). Geriatric Depression Scale (GDS): Recent Evidence and Development of a Shorter Version. Clinical Gerontologist, 5(1-2), 165-173. https://doi.org/10.1300/J018v05n01_09

Zhao, Q.-F., Tan, L., Wang, H.-F., Jiang, T., Tan, M.-S., Tan, L., Xu, W., Li, J.-Q., Wang, J., Lai, T.-J., \& Yu, J.-T. (2016). The prevalence of neuropsychiatric symptoms in Alzheimer's disease: Systematic review and meta-analysis. Journal of Affective Disorders, 190, 264-271. https://doi.org/10.1016/j.jad.2015.09.069 